\title{
Digital 3D reconstruction for the multiscale investigation on the Drawing of the fortified wall of Turin Marco Vitali
}

Politecnico di Torino - Department of Architecture and Design (DAD), Torino, Italy, marco.vitali@polito.it

\begin{abstract}
The birth in Roman times, the development in the Baroque season and the massive Napoleonic demolition of the fortified wall of Turin constitute the phases of an event still clearly readable in the current urban fabric of the historical city center: the extant elements, even if portions of a highly fragmented layout, are punctual statements of an articulated pattern of superpositions, reconnections ...

Starting from the full bibliographic, cartographic and archival documentation, this contribute aims to actualize the numerous study schemes, drawn up over decades, anchoring their course, sketched by traces and additions, to the digital cartography.

The goal is to construct a matrix from which it is possible to extrapolate thematic analysis oriented to survey and digital reconstruction of special items: city gates, bulwarks ...

The 3D representations could be used as a medium of interpretation and communication of the transformation, sedimentation and structuring of the urban form.
\end{abstract}

Keywords: Drawing, 3d modeling, virtual reconstruction, graphic analysis.

\section{Introduzione}

Le cinta fortificata della città di Torino costituisce un complesso in cui si stratificano, si sovrappongono, per conferme o smentite, tracciati riconducibili alle diverse epoche di impianto, secondo una storia comune a molti insediamenti.

Gli episodi che descrivono la nascita e lo sviluppo di questo sistema costituiscono le fasi di una vicenda ancora chiaramente leggibile nell'attuale tessuto urbano del centro storico della città, la cui lettura, passando attraverso l'individuazione e l'interpretazione dei rapporti, inscindibili, tra architettura e ambiente, sottolinea la necessità di sintesi storico critiche aperte, attente agli esiti materiali sul territorio come fenomeni in divenire. A tale scopo, al fine di inquadrare il tema e le problematiche ad esso connesse ripercorriamo sinteticamente le fasi di sviluppo di questo complesso apparato, cercando di mettere in risalto gli aspetti di carattere urbanistico e le relazioni che via via si intessono con la forma urbana, tralasciando quegli aspetti, specificatamente tecnici, dell'arte militare.

Sulla base della ricca documentazione cartografica, bibliografica ed archivistica, si sono selezionate le fasi salienti delle trasformazioni urbane connesse allo sviluppo, ammodernamento e crescita, nonché alla demolizione della cinta fortificata tratteggiate qui di seguito.

\section{Evoluzione storica del tracciato della cinta}

Il tracciato originario delle fortificazioni risale all'epoca romana (I sec. d.C.): la cinta, composta secondo lo schema classico a pianta quadrata, era costituita da mura che superavano i cinque metri di altezza e i due metri di spessore e rafforzata da cinque torri angolari ottagonali, da 
numerose torrette di guardia su ciascun lato (in corrispondenza dello sbocco delle vie cittadine) da un certo numero di postierle, posizionate presumibilmente in corrispondenza di ogni torretta. In asse alle direttrici viarie di cardo maximus (l'attuale tracciato di via San Tommaso e via Porta Palatina) e decumanus maximus (l'attuale via Garibaldi) si aprivano le quattro porte cittadine, costituite da edifici di notevoli proporzioni: Decumana, Pratoria, Principalis Dextera e Principalis Sinistra.

Contrariamente a quanto avvenne per altre città romane $^{1}$, la cinta muraria non subì modificazioni sostanziali (alcune ricostruzioni e l'aggiunta di un fossato ${ }^{2}$ fino al tempo di Emanuele Filiberto. Ciò nonostante, alle originare porte romane si erano aggiunte, nei secoli XII e XIII, almeno sei porte secondarie correlate alla nuova struttura funzionale della città: la prima a perdere in parte la propria funzione di accesso privilegiato sembra essere stata proprio la Porta Palatina (prima Principalis Sinistra, poi Doranea e ancora successivamente Palatina), a favore della più occidentale Porta Pusterla. La Porta Decumana, sostituita a partire dal 1208 dalla Porta Fibellona (un varco aperto nelle sue immediate vicinanze), nel tempo viene occupata da strutture edilizie sulle quali ha origine la fabbrica del castello, realizzato a partire dal 1317 da Filippo di Savoia-Acaia (1264-1334): le annesse opere di fortificazione vennero demolite in occasione della costruzione di una nuova cinta bastionata ai quattro angoli - esterna e parallela a quella antica - e rimpiazzate, nel 1535 , da un grande baluardo.

La Cittadella, in forma di stella pentagonale collocata in corrispondenza dell'angolo sudovest delle fortificazioni, venne realizzata, tra il 1564 e il 1566, ad opera di Francesco Paciotto da Urbino e successivamente ampliata e perfezionata.
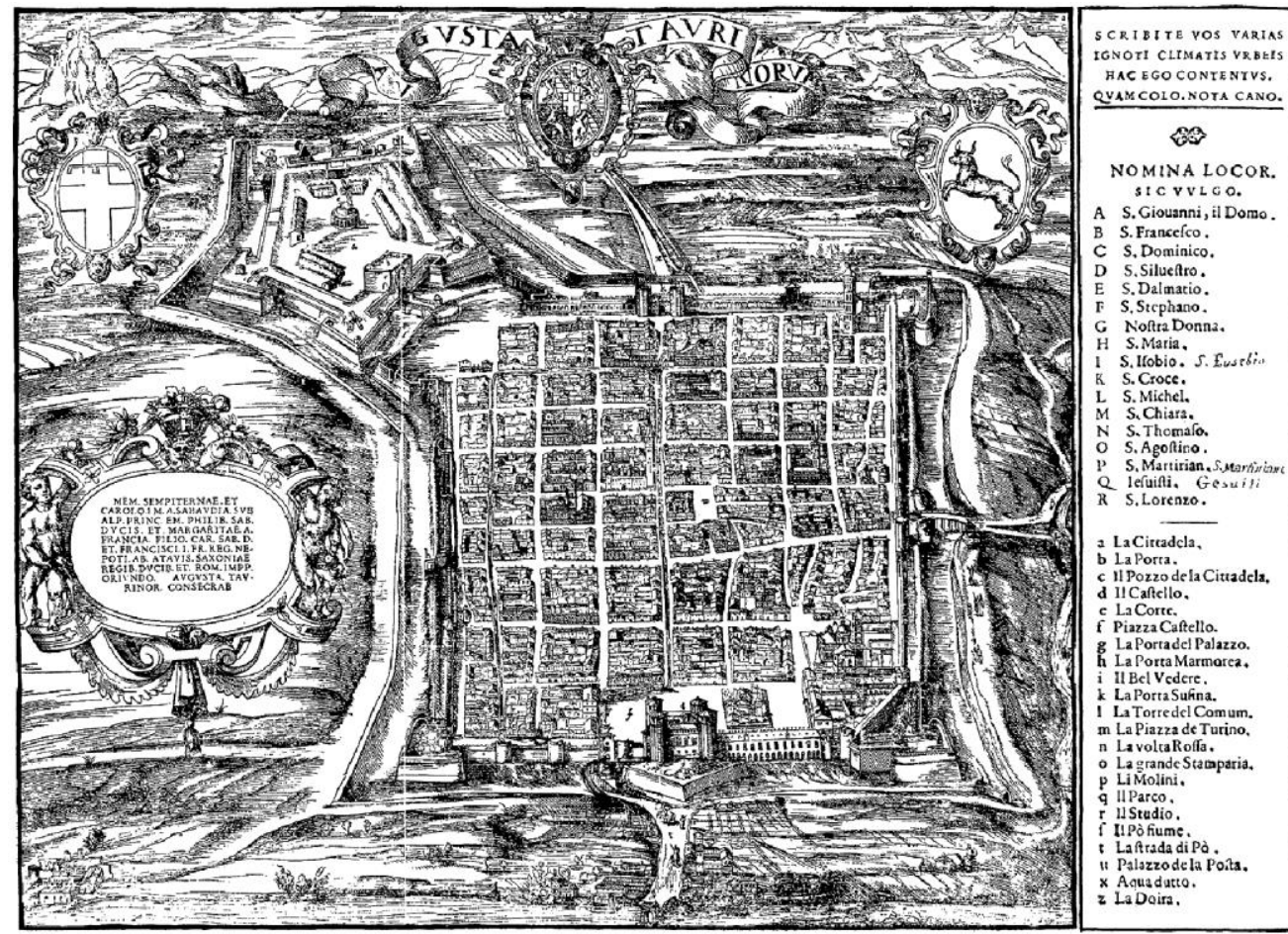

Fig. 1 - Giovanni Carracha (incisa su legno da Johann Krieger nel 1572): «PHILIBERTI / PINGONII / SABAUDI / AUGUSTA / TAURINORUM / TAURINI, Apud haeredes Nicolai Bevilaquae, MDLXXVII. 
La pianta del Carracha ${ }^{3}$ sintetizza e descrive in maniera estremamente puntuale la configurazione delle fortificazioni e del tessuto cittadino a metà "500: questa veduta "a volo d'uccello" della capitale sabauda, ricchissima di dettagli e corredata da una legenda esplicativa dei principali monumenti e luoghi cittadini, consente di riconoscere tutti gli elementi appena descritti.

Nel 1618-20 Carlo Emanuele I decide, su progetto di Carlo di Castellamonte, di spezzare la cinta quadrata con un primo ampliamento verso sud, in forma di asola fortificata, che collega la cittadella con il baluardo cinquecentesco di Santa Margherita (angolo sudest). L'espansione della città comportò l'abbattimento della porta detta Marmorea (la Principalis Dextera romana) che fu sostituita dalla Porta Nuova al termine della omonima via (attuale Via Roma); la porta verso Susa venne spostata in prossimità di un bastione della cinta muraria mentre l'antica porta Segusina (la Porta Pretoria romana) era già scomparsa nel 1585 ; anche le Porte Palatine persero la loro funzione viaria e la Porta di Palazzo venne spostata tra i due bastioni eretti sul lato nord della cinta.

Il secondo ampliamento della città, promosso da Vittorio Amedeo di Savoia nel 1680, si sviluppa sul territorio fra la città e il Po, inglobando le molte preesistenze attestate sulla via della calce: per sopperire alla necessità di spazio edificabile si abbatterono le mura romane e filibertiane che ancora dividevano la città vecchia dalla città moderna (primo ampliamento) e si realizzò nel settore est del territorio suburbano, ad opera di Amedeo di Castellamonte, una nuova ampia asola fortificata che partendo da uno dei bastioni dell'ampliamento sud (S. Cristina), giunge a lambire il Po, per andarsi a riagganciare, in corrispondenza del Bastion Verde, alla antica cinta di Emanuele Filiberto. La porta di Po (Guarino Guarini, 1679) viene costruita tra il sesto e il settimo bastione.

A partire dal 1719 si inizia l'ampliamento occidentale, ad opera di Filippo Juvarra, che, agganciandosi al cinquecentesco Bastione della Consolata, si sviluppa in tre bastioni regolari e una bretella di collegamento alla cittadella, (simmetricamente al primo tratto del primo ampliamento verso sud): tra il secondo e il terzo bastione viene aperta la Porta Susina.

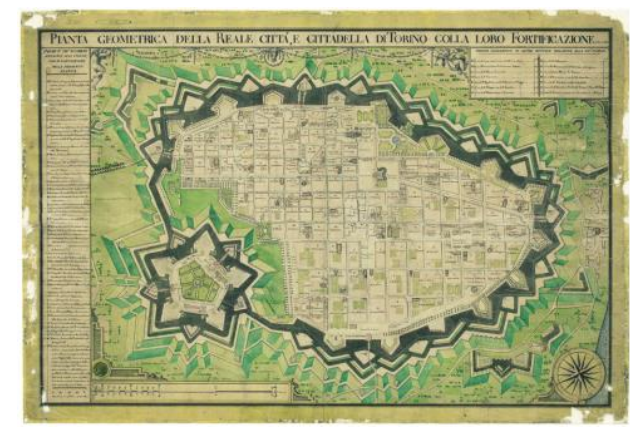

Fig. 2- Ignazio Amedeo Galletti, 1790: Pianta geometrica della reale città, e cittadella di Torino colla loro fortificazione (ASCT, Archivio Storico della Città di Torino; Tipi e Disegni, 64.2.13.)

\section{Emergenze e tracce in filigrana}

In epoca napoleonica viene deciso l'abbattimento delle mura della città, salvando solo la cittadella, le Porte Palatine, adibite a carcere militare, alcuni tratti delle mura romane ed alcuni bastioni trasformati in giardini: quello di S. Ottavio (Bastion Verde), poi demolito nel 1891, i bastioni di S. Giovanni e di S. Andrea, spianati nel 1874, parte di quello di $S$. Antonio e quelli di S. Lorenzo, S. Maurizio e S. Carlo, che, unici superstiti, sorreggono il Giardino Reale. Le mura vennero demolite in poco tempo e la città fu libera di espandersi in ogni direzione.

Ritiratisi i Francesi, con la stagione inaugurata Vittorio Emanuele I, vennero formati ed aperti gli ampi viali di circonvallazione della città, conformemente ai progetti napoleonici (attuali corso S. Maurizio, corso Regina Margherita, corso Principe Eugenio, corso Inghilterra, corso Vittorio, corso Cairoli) e le grandi piazze (piazza Vittorio Veneto, 1818; piazza Carlo Felice, 1823; piazza della Repubblica, 1837), a cui si aggiunge, dopo lo smantellamento della cittadella (dal 1852) la piazza Statuto (1865).

Nel tessuto cittadino odierno resta ben poco di tutto questo complesso sistema: infatti, oltre alle emergenze precedentemente elencate, è possibile 
segnalare solamente la presenza di parte delle mura romane, il basamento della torre angolare romana all'angolo nord ovest (in adiacenza al complesso della Consolata, qualche elemento di comunicazione e di ricetti sotterranei ${ }^{4}$, un bastione barocco e il maschio della Cittadella.

Queste sporadiche e puntuali emergenze non costituiscono da sole elementi utili al tracciamento, attuallizzato e ancorato alla cartografia attuale, dell'andamento della cinta e delle sue espansioni: tuttavia, seppure non da un punto di vista archeologico ma per lo meno da un punto di vista urbanistico, sono ancora ben visibili anomalie e irregolarità nei tracciamenti viari e nella organizzazione dei lotti che possono essere letti e presi in considerazione per fornire indicazioni fondamentali al lavoro che ci siamo proposti. Lavorando, così come appena descritto, per tracce ed integrazioni, si riesce a ricostruire abbastanza fedelmente quasi tutto il tracciato delle difese della città.

Della cinta quadrata appare evidente, in relazione a ciò che resta e ad alcuni scavi condotti (come per esempio quelli realizzati all'angolo tra gli attuali corso Siccardi e via Cernaia $)^{5}$, l'intero percorso della cinta e la posizione, abbastanza precisa, delle porte.

Più difficile e incerto risulta il tracciamento, in filigrana, dell'ultima cinta difensiva, quella seisettecentesca: questa infatti, è coglibile soprattutto dal comportamento anomalo del tracciato viario, rispetto alla scacchiera ortogonale, e dalla forma irregolare di certi isolati.

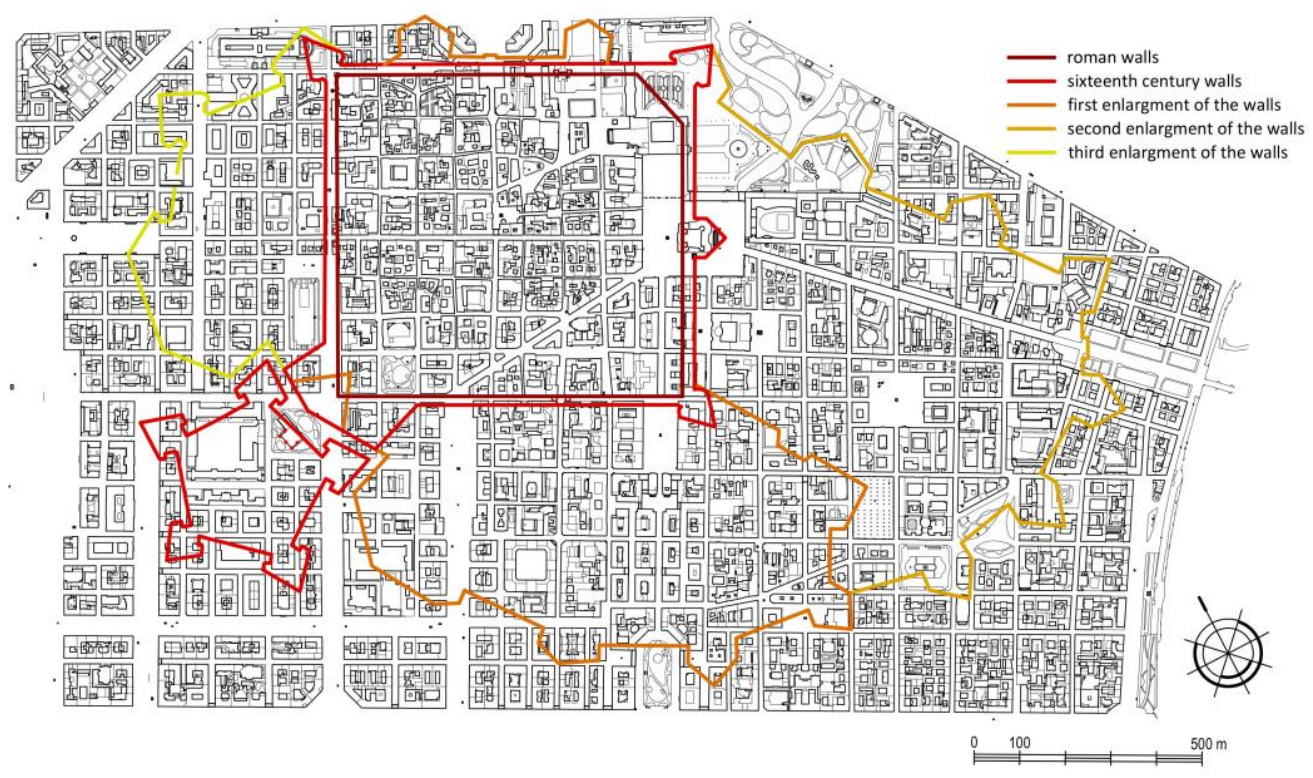

Fig. 3- Ricostruzione, sulla base digitale costituita dalla carta Tecnica del Comune di Torino, dei tracciati delle successive espansioni della cinta fortificata della città (elaborazione grafica di Marco Vitali)

Seguendo queste anomalie si riesce a ricostruire con una certa attendibilità il tracciato originario seguendo l'andamento di via Andrea Doria, passando per i giardini e la piazza Cavour, lambendo in fregio l'isolato della chiesa di S. Michele e la parte settecentesca del convitto delle Rosine, attraversando piazza Vittorio Veneto, seguendo il tracciato dell'attuale corso san Maurizio, per poi ricollegarsi al bastione di S. Lorenzo (nella sua pressoché completa integrità), seguito dalle porte Palatine, la piazza Emanuele Filiberto, la torre angolare della Consolata, la via Gaetano Valerio, i Quartieri dello Juvarra, corso Valdocco, il maschio della Cittadella, Piazza Solferino e Corso Giacomo Matteotti, che chiude l'anello. 
In questo modo, sebbene con un certo grado di approssimazione, si è potuto individuare, utilizzando come base la cartografia numerica del comune di Torino, le vestigia e le porzioni attualmente ancora esistenti e, sulla base delle considerazioni e delle interpretazioni appena esposte, tracciare le porzioni mancanti del disegno complessivo, attualizzando i numerosi schemi di studio e lettura redatti nel corso dei decenni.

\section{Possibili sviluppi della ricerca}

Questa cartografia digitale tematica costituisce, almeno nelle nostre intenzioni, una base omogenea per studi e approfondimenti puntuali successivi, che ne possano definire localmente, con un maggiore livello di affidabilità e precisione, andamenti, tracciati e consistenze.

Il fine è di costituire una matrice a partire dalla quale estrapolare approfondimenti tematici orientati al rilievo dell'esistente, alla ricostruzione digitale di particolari elementi, come porte, bastioni, ecc. In tal senso la rappresentazione tridimensionale potrebbe, in questo contesto, essere utilizzata come mezzo di interpretazione e comunicazione della trasformazione, sedimentazione e strutturazione della forma urbana per letture diacroniche, sequenziali, nonché come strumento di analisi, confronto e verifica delle fonti, un contenitore che si struttura attraverso la lettura e l'organizzazione di documenti disomogenei: disegni di progetto - realizzati e non rappresentazioni qualitative, vedute, fotografie...

A titolo esemplificativo si è pensato di strutturare, almeno nel reperimento della documentazione necessaria e nelle possibili elaborazioni da progettare e impostare metodologicamente, un lavoro di approfondimento tematico che si concentra su una porzione contenuta, ma criticamente selezionata della cinta, in cui le vestigia costituiscono emergenze di notevole prestigio: si tratta della porzione a cavallo delle Torri Palatine.

Si tratterebbe infatti di mettere in relazione una mole considerevole di dati, a partire dai rilievi storicamente condotti durante il XVII secolo ${ }^{6}$ ed in occasione dell'importante restauro realizzato ad opera di d'Andrade ${ }^{7}$, della cartografia storica sia a scala urbanistica (tutti i piani relativi al governo della città), dei documenti di addeguamento e progetto redatti nel corso dei secoli XVII e XVIII (si pensi, per esempio, ai documenti settecenteschi relativi alle

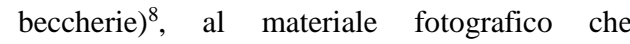
testimonia lo stato dei luoghi prima della realizzazione dei pesanti interventi di restauro sopra citati, dei lavori di studio e rilievo operati in ambito accademico ${ }^{9}$, dei più recenti lavori di restauro ${ }^{10}$, delle grandi opere condotte dalla municipalità $^{11}$, e ancora, cambiando scala di studio, alle interessanti relazioni che cinta fortificata $e$ tessuto urbano intessono in occasione delle grandi trasformazioni che hanno coinvolto le aree in adiacenza (i terreni su cui è stata costruita la porta di ingresso alla città di Porta Palazzo e la risistemazione dell'area mercatale in espansione, e ancora più in la', la piazza Emanuele Filiberto, con l'ampliamento dell'edificato in fregio alle mura romane).

Tale porzione raccoglie in sé, come abbiamo appena brevemente esposto, una variabilità di stratificazioni che consente di considerarla campione per la valutazione di un metodo di indagine che studi l'allestimento dei supporti tecnologici per la realizzazione degli approfondimenti alle diverse scale, indaghi le possibili relazioni e connessioni tra gli esiti grafici e gli strumenti digitali di modellazione, nonché le importanti ricadute metodologicodisciplinari per l'avanzamento e l'affinamento della ricerca. 

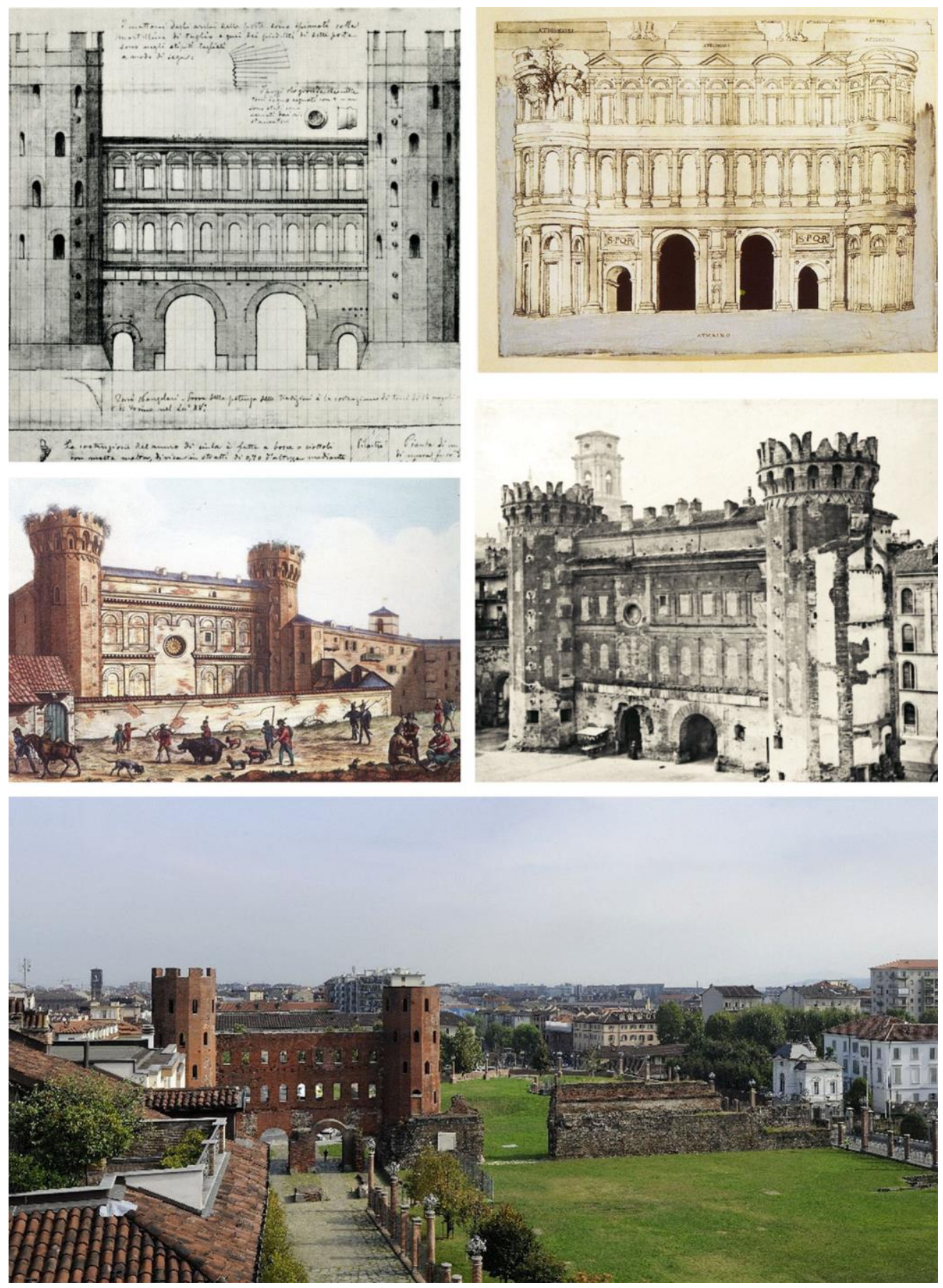

Fig. 4-. Dall'alto a sinistra: Alfredo d'Andrade, Rilievo della porta (1883); Giuliano da Sangallo, rilievo congetturale di una delle porte della cinta; veduta prospettica ottocentesca delle torri; la Porta palatina dopo la demolizione degli edifici addossati e i restauri della seconda metà del XIX sec.; foto attuale. 


\section{Notes}

(1) Cfr. Cavallari Murat (1968), p.877.

(2) Dell'esistenza del fossato in epoca medievale e dei ponti levatoi, infatti, si fa ripetutamente cenno negli statuti della città, così come riportato dal Cavallari Murat (cit.), p. 878.

(3) Questa pianta, voluta da Emanuele Filiberto di Savoia e incisa su legno da Johann Krieger nel 1572 su disegno di Giovanni Carracha, rappresentò un'assoluta novità: le raffigurazioni sino ad allora realizzate erano del tutto imprecise, sommarie, spesso idealizzate, e riportavano solo il perimetro cittadino senza altra pretesa descrittiva; non a caso questa mappa resterà il prototipo per le piante a venire per circa un secolo. Sull'angolo sud-occidentale della città si impone la poderosa mole della cittadella voluta da Emanuele Filiberto, nucleo della futura città fortificata e, per Torino, unica opera architettonica di rilievo del Cinquecento.

(4) Per quanto riguarda la rete di gallerie e passaggi sotterranei, parte di essi sono visitabili nel museo Pietro Micca, da cui si può accedere ad alcuni elementi ancora conservati delle antiche fortificazioni. Lo sviluppo urbanistico della città ha cancellato quasi completamente le fortificazioni ma ha lasciato pressoché intatta la rete delle gallerie (il cui sviluppo stimato è di circa $14 \mathrm{~km})$, di cui $9 \mathrm{~km}$ sono ancora attualmente percorribili.

(5) Cfr. Cavallari Murat (1968), pp. 898-900.

(6) A tal proposito si vedano i disegni del "Rilievo ottocentesco dell'edificio inglobante le romane Porte Palatine. G. Barone, 1851. Sei disegni a penne nera e rossa, acquerellati in grigio, rosso e arancio (Archivio di Stato di Torino, AST, sezione $2^{\circ}$, Demani, Elenco 3 Pacco 5, Div. VI, versamento 18866. N. 51.

(7) Nei primi anni del Novecento si decide di procedere alla completa liberazione del delle Portr Palatine, demolendo tutte le strutture posteriori e scavando l'area del cavaedium. Il progetto viene avviato da Alfredo D'Andrade (Alfredo Cesare Reis Freira de Andrade, Lisbona 26 agosto 1839 - Genova, 30 novembre 1915), architetto e direttore dell'Ufficio
Regionale per la Conservazione dei Monumenti del Piemonte e della Liguria, che mira a cancellare gli interventi di restauro precedenti. I lavori vanno avanti a lungo e in maniera discontinua fino allo scoppio della prima Guerra Mondiale. Nel 1935 si avvia un nuovo cantiere di restauro, ma anche questa volta i lavori, nel corso di molti decenni, non giungono a conclusione.

(8) A tal riguardo si possono vedere i Disegni in pianta delle botteghe per il quartiere dei macelli di Porta Palazzo, in coerenza alle Torri di quell'antica Aorta, 1724. ASCT, Carte sciolte, n. 4719; e ancora, Gioachino Butturini, Casa propria della città di Torino posta nell'Isola di S. Marziale denominata de' Macelli / camere del P.mo piano / Piano delle Botteghe / Piano delle Crotte, 1789. ASCT, Collezione X, 22-25.

(9) Ardissone P.; Bornaz L.; Lo Turco M.; Vitali M. (2005).

(10) Papotti L. (2003), "La Porta Palatina. L'intervento di restauro degli anni novanta". In Mercando L. (a cura di), Archeologia a Torino. Dall'età preromana all'alto Medioevo. Allemandi. Torino. pp. 89-96.

(11) Il parco archeologico della Porta Palatina costituisce uno degli interventi principali di una strategia promossa fin dagli anni Ottanta del secolo scorso dal Ministero per i beni Culturali, in sinergia con l'amministrazione cittadina per la riqualificazione dell' area romana.

Il progetto di Giovanni Durbiano, Luca Reinerio, Aimaro Isola ed Egidio Cupolillo disegna un parco che sfrutta il dislivello di circa $4 \mathrm{~m}$ tra piazza San Giovanni e corso Regina Margherita, creando un unico piano che da piazza $S$. Giovanni si estende oltre le mura romane innalzandosi a formare un bastione ispirato alle fortificazioni seicentesche, demolite a partire dall'epoca napoleonica. Gli spazi ricavati al di sotto del nuovo "bastione" sono stati progettati per la rimessa delle strutture mobili del mercato di Porta Palazzo.

A tal proposito si vedano le pubblicazioni:

Oreglia d'Isola A., Durbiano G., Reinerio L. (2004), "Il parco archeologico di Torino". In Il 
Giornale dell'architettura. A. III, n. 20. Torino. Oreglia d'Isola A., Durbiano G., Reinerio L. (2006), "Il progetto del Parco archeologico delle
Torri Palatine: valori e condizioni di un luogo", in Studi piemontesi. A. XXXV, n. 2. Torino. pp. 287-300 